\title{
Influence Among Preferences and Its Transformation to Behaviors in Groups
}

\section{An Agent-Based Modeling and Simulation of Fertility Intention and Behavior}

\author{
Hang Luo $\mathbb{D}$, Zhenjie Wang ${ }^{(\varpi)}(\mathbb{D}$, Shengzi Yang $(\mathbb{D}$, Hanmo Yang (D), \\ and Yuke Gong (D) \\ Peking University, Beijing 100871, China \\ \{hang. luo, zhenjie. wang, ysz, hanmo. yang, \\ gongyuke\}@pku.edu.cn
}

\begin{abstract}
We consider settings of group decision and negotiation where agents' preferences (such as intentions, beliefs and opinions) are influenced by each other and thus their behaviors are possibly changed. We build a multi-agent system $\left(\mathrm{MAS}_{\text {IITIB }}\right)$ in the context of social networks to model the mutual influence among agents' intentions and the transformation from agents' intentions to their behaviors in groups. On the micro level of individual agents, we construct the self-evolution rule of agents' intentions and the generation, constraint and termination rule of agents' behaviors; on the macro level of networked structure, we describe the mutual influence on intentions among agents, which can be diversified in both strength and polarity. We detail this multi-agent system and run experiments and simulations using the interaction of fertility intentions and the generation of fertility behaviors in families as example. Two experimental programs are designed: one is to adjust the initial fertility intentions of prospective parents, and the other is to adjust the range of weight of influence among family members, to investigate the effects on the childbearing behavior and the number of newborn children in the long-term. This study intends to provide modeling bases for the dynamics of preferences and behaviors due to mutual influence among agents in groups, particularly for the study of fertility intention and behavior in families, and more broadly, the forecast of population growth and effects of fertility policy. It is an innovative try of distributed artificial intelligence (multi-agent system) in the field of demography and public policy, and provides with a new bottom-to-up perspective and unconventional agent-based method.
\end{abstract}

Keywords: Influence among preferences - Transformation from preferences to behaviors $\cdot$ Agent-based modeling and simulation $\cdot$ Fertility intention $\cdot$ Fertility behavior 


\section{Introduction}

In the context of multi-agent systems (such as group decisions and negotiations), the influence among agents' preferences (such as intentions, beliefs, opinions) and behaviors is quite common and has been discussed by scholars from varied disciplines $[20,21]$, including computer science (particularly artificial intelligence and multi-agent system) [4, 17, 20, 21, 23, 24, 26, 27], economics, decision theory, and social networks [5-9, 12-16]. During the process of a group decision or negotiation, an agent usually has full motivation to influence others' preferences or behaviors with his/her own preferences or behaviors, to make them believe what he/she believes, behave as what he/she behaves, thus increasing the possibility that his/her preference or behavior becomes the mainstream of the group, and gaining more utility and satisfaction [20].

\section{Influence on Preferences Among Agents and Its Transformation to Behaviors in Multi-agent Systems}

\subsection{Transformation from Preferences to Behaviors}

In a multi-agent system, though influencing and influenced with each other, every agent's behavior is (eventually) dependent on his/her own preference, no one can be forced to adopt a behavior against his/her own willingness. Actually, this is a fundamental feature of a multi-agent system (such as a group decision and negotiation) where there is no agent as an (absolute) authority who can command and control other agents' behaviors [19]; thus, influencing an agent's preference is the precondition of influencing his/her behavior. Therefore, in the context of a group decision and negotiation, not only the mutual influence among agents' preferences should be discussed, but also the transformation from agents' influenced preferences to their behaviors needs to be investigated. In fact, an influenced (updated) preference will possibly lead to the adoption of a (new) behavior, or not [21]. There is randomness due to bounded rationality and changing environment. The conditions for the generation of behaviors under influence is an important issue that deserves full discussions.

\subsection{Weighted and Signed Influence Among Preferences}

In real-world settings, the influence faced by one agent usually comes not from one agent at a time, but from more than one agent at the same time [20, 21]. What's more, the multiple influences are usually diversified in both polarity and strength, such as a positive influence from a friend (or ally) versus a negative influence from an enemy (or opponent) $[19,20]$ and a strong influence from a close friend (or family, or relative) versus a weak influence from an ordinary friend [20,21]. Even in a group with members close to each other such as a family, influences from different members can also be distinguished by strength and even polarity (as not all relations in a family are harmonious at all times). 


\section{Strength of Influence}

The strength of influence between two agents can be affected by many factors, for instance: (1) the actual or perceived power (or force, or authority) of the influencer from the perspective of the influenced one, considering that the bigger the power of the influencer is, the greater is the weight of the influence [20]; (2) or how much the influenced one trusts (or believes) the influencer, considering that the higher the trust is, the greater is the weight of the influence [17]; (3) or the similarity of preferences between the influencer and the influenced one, considering that the more similar their preferences are, the greater is the weight of the influence, as people are usually inclined to listen to the opinions that are same and resist the opinions that are different from their own.

\section{Polarity of Influence}

The polarity of influence between two agents can also be affected by many factors, for instance: (1) the closeness of relationship between the influencer and the influenced one, considering that the closer the relationship is, the more possible it is for the influencer to exert a positive influence on the influenced one; (2) or the compatibility of objectives (or purpose, or interests) between the influencer and the influenced one, considering that the more contradictive their objectives are, the more possible it is for the influenced one to receive a negative influence from the influencer.

\section{An Example of Influence Among Preferences and Behaviors in Groups: Fertility Intention and Behavior in Families}

To model the mutual-influence among agents' preferences and the transformation from agents' preferences to their behaviors in groups with details of practical issues, we choose the case of fertility intention and behavior in families. In fact, a family is a very typical group composed of more than one member, with abundant group behaviors and full of constant communications among members. The interactions among family members consist of typical group decisions and negotiations. Particularly in a "democratic" instead of "dictatorial" family, all family members collectively make decisions and ceaselessly negotiate on critical family issues, such as whether and when to bear a child. This kind of decision will profoundly change the life of a family in the long-term, thus, all family members will join the process of decision-making and usually all try their best to convince and influence other members with their own preferences in order to achieve a desirable result. For example, a couple does not want to bear a child, but the couple's parents may have strong intentions to have a grandchild and will try to convince the couple from time to time; on the other side, the couple may resist their parents" "traditional" opinions and even try to sell their own "modern" ideas to their parents. Therefore, the case of fertility intention and behavior in families could be a perfect example to study the mutual influence among preferences and its transformation to behaviors in groups. 


\section{From Individual Intention, to Individual Behavior and to Social Evolution: A New Approach for Demography}

The fertility rate and population growth have always been a critical issue in human society. Particularly in China, families' reproductive behaviors are profoundly affected and constrained by the population and fertility policies. The well-known fertility policy in China was simplified as the "one-child policy", which had been strictly enforced on childbearing behaviors (especially of urban residents) for more than three decades [10, 28]. Under the one-child policy, $36 \%$ of the population strictly had only one child; $53 \%$ of the population were permitted to have a second child if their first child was a girl; $9.6 \%$ of the population were permitted to have two children regardless of their first child's gender; and $1.6 \%$ (mainly ethnic minority) had no limit at all [3]. It implies an average of 1.47 children per couple [11], falling far under the international population replacement rate of 2.1 (which means the population of China will be decreasing fast in the future). The tremendous reduction in fertility rate, together with the improved life expectancy and other demographic and socioeconomic factors, has forced China to face a more rapid and serious challenge of population aging than other countries (particularly the so-called "aging before getting rich"1).

From January 1st, 2016, China officially abandoned the one-child family planning policy, allowing all couples in China to have two children regardless of their household registration type (i.e., urban or rural hukou), region, ethnicity, and sibship size [25]. The new policy is called the "universal two-child policy". Because of this major change of fertility policy, approximately an additional 90 million women have become eligible to have the second child [31]. The implications of the universal two-child policy are of great public interest and policy concern. However, the fertility intention and actual fertility behavior may significantly differ from what is intended by the policy. A few studies have estimated the long-term effects of this new policy on fertility level and population aging $[30,32]$. Till now, the actual fertility rate under the new policy has been much lower than the government's expectation.

Research on population prediction can be traced to Professor King who built a simple mathematical model and calculated manually in $17^{\text {th }}$ century. Then, Malthus put forward the Malthus growth model based on the assumption of a stable population growth rate. Moreover, Verhulst proposed Logistic block population growth model, which could better describe the variation of population growth [2, 18, 29]. Leslie came up with a comprehensive population prediction model, the well-known Leslie Matrix model, containing multiple factors [22]. Subsequently, an innovative population optimal model that accurately describes the dynamic changes of people's ages was developed by Andrew and Meen [1].

However, all the above studies started mainly from a macro perspective of entire system, focusing on a whole country or a whole society, nearly no work has started from a micro perspective of individual interactions, particularly from the perspective of

\footnotetext{
${ }^{1}$ It means that China has faced a serious aging problem which is more common in developed countries, while China still as a developing country is not rich like other aging societies such as Germany and Japan yet.
} 
members in a family, explored how family members' interactions and mutual influences affect couples' childbearing behaviors. This paper introduces an agent-based modeling and simulation approach for the study of fertility behavior and population growth. It is based on a set of intelligent and autonomous agents that can communicate, interact and influence with each other. Each agent represents an individual member of a family and a multi-agent system represents the interaction among family members. In each interaction, every agent's preference (namely, a family member's fertility intention) is influenced by his/her own and other agents' preferences (namely, other family members' fertility intentions). Under certain conditions based on fertility intentions, a fertility behavior of the family will be produced (namely, a child to be born).

\section{Mathematical Model: Variables Definition and Rules Design}

We build the mathematical model of a multi-agent system with the mutual influence among agents' intentions and the transformation from agents' intentions to their behaviors $\left(\mathrm{MAS}_{\mathrm{IITIB}}\right)$, using the case of the interaction among fertility intentions and the generation of fertility behaviors in families, define variables and design rules in the model as follows:

\subsection{Define Variables}

$A n_{(i)}(t)$ represents agent $i$ (namely, family member $i$ ) at time $t$ (namely, $t$-th mutual influence among family members), $i \in \mathbb{N}=\{0,1,2, \ldots, n-1\}, \mathbb{N}$ represents the set of all family members (assume there are $n$ members in a family), where 0 means the prospective mother, 1 means the prospective father, and so on; $t=1,2, \ldots$ represents the increasing time (the unit of time is set as one month ${ }^{2}$ ) since the beginning of simulation. The attributes of individual agents, characteristics of relational links (namely, influences) between agents, and the variables of the environment are defined as follows:

(1) Intention $_{(i)}(t)$, abbreviated as $I_{(i)}(t)$, represents family member $i$ 's fertility intention at time $t$. Set $I_{(i)}(t)=x[-1 \leq x \leq 1]^{3}$, the higher a member's fertility intention is, the more this member inclines to bear a child or hopes his/her family to bear a child. $I_{(i)}(t)<0$ means he/she does not want his/her family to bear a child, $I_{(i)}(t)>0$ means he/she expects his/her family to bear a child;

(2) Initial-Age $(0)$, abbreviated as age $_{(0)}$, represents the prospective mother's initial age at the beginning of simulation. Set $a g e_{(0)}=x[180 \leq x \leq 588]$, as the childbearing age of women is usually between 15-49 years (namely, 180-588 months) old;

\footnotetext{
${ }^{2}$ The unit of time is set as one month as a woman ovulates one time every month, thus, having one chance to bear a child every month. Besides, one month is long enough for a full communication, interaction and mutual influence among family members.

${ }^{3}$ A random floating number is given between -1 and 1 .
} 
(3) Age-of-Peak-of-Intention $(0)$, abbreviated as $a g e_{(0)}^{P}$. The intention of a woman to bear a child is usually affected by her age. Set $\operatorname{ag} e_{(0)}^{P}=x[300 \leq x \leq 408]$, as women's fertility intentions usually peak between 25-34 years (namely, 300-408 months) old. When she is younger than certain age, her fertility intention will increase with time, but when she is older than certain age, her fertility intention will decrease with time;

(4) Behavior $_{(0)}(t)$, abbreviated as $B_{(0)}(t)$, represents the prospective mother's fertility behavior at time $t$. Set $B_{(0)}(t) \in\{0,1\}, 0$ means no child is born at time $t, 1$ means one child is born at time $t$;

(5) weight $_{(j, i)}(t)$, abbreviated as $w_{(j, i)}(t)(i, j \in \mathbb{N})$, represents the weight of influence from family member $j$ to family member $i$ at time $t$. Family members' preferences (such as intentions, beliefs and opinions) are usually influenced by each other constantly. The influences can be stronger or weaker in strength and positive or negative in polarity [19-21] due to the complicated relations in families in realworld (we have to admit that not all relations among all family members at all times are harmonious). Set $w_{(j, i)}(t)=x[-1 \leq x \leq 1], w_{(j, i)}(t)<0$ means a negative (influencing) relationship between two family members (the more the influencer inclines to have a child in his/her family, the less the influenced one inclines), and $w_{(j, i)}(t)>0$ means a positive (influencing) relationship between two family members (the more the influencer expects to have a child in his/her family, the more the influenced one expects either), and the higher the absolute value of the weight is, the stronger is the influence.

\subsection{Design Rules}

\section{Intention Self-evolution Function}

Assume that the prospective mother's fertility intention will be mainly affected by her age. When she is younger than her age of peak of intention, her fertility intention will increase with the growth of her age; but when she is older than her age of peak of intention, her fertility intention will decrease with the growth of her age.

$$
I_{(0)}^{\prime}(t)=\left\{\begin{array}{l}
I_{(0)}(t-1)+x[0<x \leq y], t+\operatorname{age}_{(0)}<a g e_{(0)}^{P} \\
I_{(0)}(t-1)-x[0<x \leq y], t+\operatorname{age}_{(0)} \geq \operatorname{age} e_{(0)}^{P}
\end{array}\right.
$$

In which, $I_{(0)}^{\prime}(t)$ means the prospective mother's fertility intention after affected by her ager at time $t, x[0<x \leq y]$ means a random value between $0-y, y$ means the maximum of change of fertility intention due to the growth of age $\mathrm{a}^{4}$.

\section{Intention Mutual-influence Function}

Assume that family member $i$ 's fertility intention for his/her family to bear a child on current time $(t)$ will be influenced by other family members' and his/her own intention

\footnotetext{
${ }^{4}$ We preliminarily set $y$ as 0.1 in the simulation.
} 
on former time $(t-1)$, as it is really hard for people to avoid the influence from their own family members.

$$
I_{(i)}(t)=\frac{\sum_{j \in \mathbb{N} \backslash\{0\}} w_{(j, i)}(t-1) I_{(j)}(t-1)+w_{(0, i)}(t-1) I_{(0)}^{\prime}(t)}{\sum_{j \in \mathbb{N} \backslash\{0\}}\left|w_{(j, i)}(t-1)\right|+\left|w_{(0, i)}(t-1)\right|} \quad i=0,1,2, \ldots, n-1
$$

We assume that the prospective mother's fertility intention that is influencing is different from other family members', as it is the prospective mother's fertility intention after affected by her age $\left(I_{(0)}^{\prime}(t)\right)$ that is influencing her own and her family members' fertility intentions.

\section{Behavior Generation Function}

Assume when both the prospective mother and prospective father's fertility intentions are high enough, a childbearing behavior will be motivated, and a child will be born. Both prospective mother and father's fertility intentions are normalized values $(x[-1 \leq x \leq 1])$ and are used as probabilities for the generation of a fertility behavior when they are positive. Thus, the higher both of prospective mother and father's fertility intentions are, the more possible it is for them to bear a child.

$$
\text { If } \begin{gathered}
I_{(0)}(t)>x[0 \leq x<1] \wedge I_{(1)}(t)>x[0 \leq x<1] \\
\text { Then } B_{(0)}(t+x[\alpha \leq x \leq \beta])=B_{(0)}(t)+1
\end{gathered}
$$

In which, $x[0 \leq x<1]$ means a random value between $0-1$. Thus, only when both prospective mother and father's fertility intentions are positive (namely, both of them are willing to have a child), there will be a possibility for the formation of a childbearing behavior. Besides, the fertilization and pregnancy of a child needs certain length of time. The time is assigned by a random value between $\alpha-\beta$ months ${ }^{5}$.

\section{Behavior Constraint Function}

Assume when the mother has just given birth to a child, her fertility intention will drop to a negative value, and cannot give birth to the next child within a certain range of time, according to the natural law.

$$
\begin{gathered}
\text { If } B_{(0)}(t)>B_{(0)}(t-1) \\
\text { Then } I_{(0)}(t)=x[-1 \leq x<0] \wedge B_{(0)}(t+x[\gamma \leq x \leq \delta])=B_{(0)}(t)
\end{gathered}
$$

After the birth of a child, the physical recovery and lactation also need certain length of time before the feasibility of the next pregnancy. The time is assigned by a random value between $\gamma-\delta$ months ${ }^{6}$.

\footnotetext{
${ }^{5}$ We preliminarily set $\alpha$ as 10 and $\beta$ as 12 in the simulation.

${ }^{6}$ We preliminarily set $\gamma$ as 10 and $\delta$ as 18 in the simulation.
} 


\section{Behavior Termination Function}

Assume when the age of the prospective mother has reached certain months $\varepsilon,{ }^{7}$ she cannot give birth any more, then the simulation stops.

$$
\text { If } t+\operatorname{age}_{(0)}>\varepsilon \text { Then Stop }
$$

\section{Computer Model}

The computer model is constructed based on the above mathematical model (MAS IITIB $_{\text {). }}$. The Netlogo ${ }^{8}$ is chosen as the implementation platform for the simulation of mutual influence among agents' preferences and the transformation from agents' preferences to their behaviors in groups, particularly the interaction of fertility intentions and the generation of fertility behaviors in families. The $3 \mathrm{D}$ evolutional view of the multi-agent system is presented in Fig. 1. We designed a networked graph to describe the relational structure among family members, with every node/agent representing a family member ${ }^{9}$ (including prospective parents, prospective parents' parents, prospective parents' relatives and friends ${ }^{10}$ ) and each link/tie representing an influencing relation between two family members. All agents interact and influence with each other in multi-periods, collectively constructing the dynamics of fertility intentions and behaviors in a family.

About the setting of structure of social networks in a family as shown in Fig. 1, it is not a complete network as a whole because there are $n$ members but there are not necessarily $C_{n}^{2}$ links among them; however, there should be several sub complete networks within the whole network according to common sense, for example: the set of \{prospective father, prospective mother, prospective father's father, prospective father's mother, prospective mother's father, prospective mother's mother should constitute a complete network which is full of all possible links, but it is not necessary to exist a link (namely, an influencing relation) between the prospective mother's relatives, friends and the prospective father.

\section{Simulation Experiments and Results}

The aim of our experiments is to simulate the mutual influence among fertility intentions and the transformation from fertility intentions to fertility behaviors in a micro individual perspective of family members, different from the conventional macro entire perspective of social systems in Demography, to investigate the long-term effects of the

\footnotetext{
${ }^{7}$ We preliminarily set $\varepsilon$ as 588 (namely, 49 years) old.

${ }^{8}$ There are several platforms such as the Swarm, Repast, Ascape, AnyLogic, Matlab for agent-based system modeling and simulation, in which, the Netlogo is notable for its ease of use and friendly interface.

${ }^{9}$ We use agents' colors to distinguish family members' genders: a blue agent indicates a male and a pink agent indicates a female.

${ }^{10} \mathrm{We}$ assume the influence from a friend is usually positive.
} 


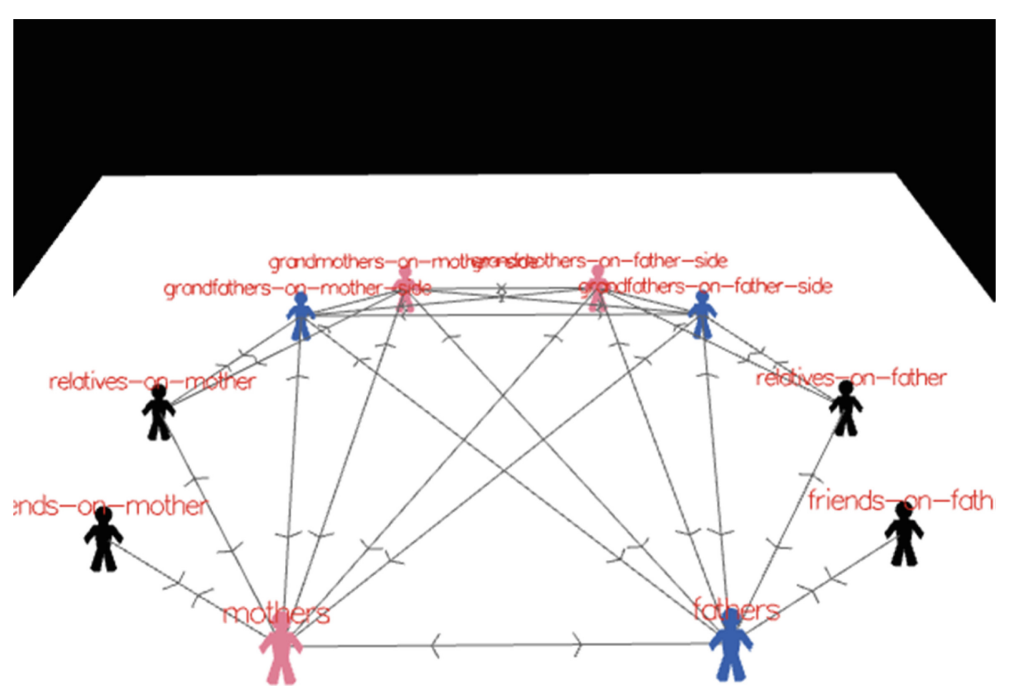

Fig. 1. Networked structure of family members in a household

initial fertility intentions of couples and the relational structure of family members on the fertility rate and population growth, particularly under consideration of the fertility policy changed from the one-child policy to the universal two-child policy in China. We design two experimental programs for computational simulations as follows:

- In experimental program I, we preliminarily set three levels for couples' initial fertility intentions, including: (1) for a low fertility intention, a random value is given from $x[-1 \leq x<0]$; (2) for a medium fertility intention, a random value is given from $x[-0.5 \leq x \leq 0.5]$; (3) and for a high fertility intention, a random value is given from $x[0<x \leq 1]$. If both the prospective father and mother's initial fertility intentions have three levels of variations, then the combinations will be $9=3 \times 3$, corresponding to 9 experimental plans. We also set a control group where we run the simulation without any intervention on the range of initial fertility intentions, which means a random value is given from $x[-1 \leq x \leq 1]$. Then, we run different experimental plans to simulate their effects on the generations of fertility behaviors and the number of new born children.

- In experimental program II, we set the range of the weight of influence for three categories of relationships between family members, respectively: between the prospective mother and the prospective father of a couple, between a prospective father/mother and his/her own parents, and between a prospective father/mother and his/her parents-in-law. We preliminarily set two levels for each category of relationships, including: (1) for a great relation, a random value of $x[0 \leq x \leq 1]$ is given to the weight of influence, which means the influencing relation must be positive; (2) for a normal relation, a random value of $x[-1 \leq x \leq 1]$ is given to the weight of 
Table 1a. Experiment I's outcomes of families' fertility behaviors under adjusting couples' initial fertility intentions

\begin{tabular}{|c|c|c|c|c|c|}
\hline \multirow[t]{2}{*}{ Variable } & \multicolumn{5}{|c|}{ Experimental outcome: numbers of families (percent of families) } \\
\hline & Control group & $a$ & $b$ & $c$ & $d$ \\
\hline \multicolumn{6}{|l|}{ No. of children (2 years) } \\
\hline 0 & $4062(40.6)$ & $4116(41.2)$ & 4058 (40.6) & $4125(41.3)$ & $4089(40.9)$ \\
\hline 1 & $3730(37.3)$ & $3743(37.4)$ & $3748(37.5)$ & $3707(37.1)$ & $3704(37.0)$ \\
\hline 2 & $2208(22.1)$ & $2141(21.4)$ & $2194(21.9)$ & $2168(21.7)$ & $2207(22.1)$ \\
\hline \multicolumn{6}{|l|}{ No. of children (5 years) } \\
\hline 0 & $3388(33.9)$ & $3379(33.8)$ & $3386(33.9)$ & $3321(33.2)$ & $3361(33.6)$ \\
\hline 1 & $3058(30.6)$ & $3181(31.8)$ & $3135(31.4)$ & $3228(32.3)$ & $3184(31.8)$ \\
\hline 2 & $3554(35.5)$ & $3440(34.4)$ & $3479(34.8)$ & $3451(34.5)$ & $3455(34.6)$ \\
\hline \multicolumn{6}{|l|}{ No. of children (lifetime) } \\
\hline 0 & $193(1.9)$ & $203(2.0)$ & $193(1.9)$ & $199(2.0)$ & $198(2.0)$ \\
\hline 1 & $1791(17.9)$ & $1806(18.1)$ & $1808(18.1)$ & $1733(17.3)$ & $1776(17.8)$ \\
\hline \multirow[t]{2}{*}{2} & $8016(80.2)$ & $7991(79.9)$ & $7999(80.0)$ & $8068(80.7)$ & $8026(80.3)$ \\
\hline & \multicolumn{5}{|c|}{ Mean (standard-deviation) } \\
\hline $\begin{array}{l}\text { Interval time between the } \\
\text { first child and the second } \\
\text { child (years) }\end{array}$ & $7.21(5.05)$ & $7.27(5.07)$ & $7.35(5.18)$ & $7.36(5.12)$ & $7.38(5.18)$ \\
\hline
\end{tabular}

Notes: control group: without any intervention;

$a$ : prospective father: high fertility intention, prospective mother: high fertility intention;

$b$ : prospective father: high fertility intention, prospective mother: medium fertility intention;

$c$ : prospective father: high fertility intention, prospective mother: low fertility intention;

$d$ : prospective father: medium fertility intention, prospective mother: high fertility intention.

influence, which means the influencing relation could be positive, but also negative, namely, harmonious or stormy. If all three kinds of relations have above two situations, then the combinations will be $8=2 \times 2 \times 2$, corresponding to 8 experimental plans. Then, we run different experimental plans to simulate their effects on the generations of fertility behaviors and the number of new born children.

For each of experimental plans, we run 10,000 times (representing the simulation of 10,000 families), and in each run, the experimental variable is given value randomly according to a certain range as we designed. In each run and in each mutual influence in it, the current intention (at time $t$ ) of one family member is affected and determined by his/her own and other family members' former intentions (at time $t-1$ ).

In the Table 1a and Table 1b, we present the outcomes of experimental program I. More than $40 \%$ of households have no child in the end of the two-year simulation. In 
Table 1b. Experiment I's outcomes of families' fertility behaviors under adjusting couples' initial fertility intentions

\begin{tabular}{|c|c|c|c|c|c|}
\hline \multirow[t]{2}{*}{ Variable } & \multicolumn{5}{|c|}{ Experimental outcome: numbers of families (percent of families) } \\
\hline & e & $f$ & $g$ & $h$ & $i$ \\
\hline \multicolumn{6}{|l|}{ No. of children (2 years) } \\
\hline 0 & 4160 (41.6) & $4062(40.6)$ & $4192(41.9)$ & $4182(41.8)$ & $4066(40.7)$ \\
\hline 1 & $3744(37.4)$ & $3827(38.3)$ & $3723(37.2)$ & $3692(36.8)$ & $3756(37.6)$ \\
\hline 2 & $2096(21.0)$ & $2111(21.1)$ & $2085(20.8)$ & $2136(21.4)$ & $2178(21.8)$ \\
\hline \multicolumn{6}{|l|}{ No. of children (5 years) } \\
\hline 0 & $3447(34.5)$ & $3321(33.2)$ & $3440(34.4)$ & $3402(34.0)$ & $3340(33.4)$ \\
\hline 1 & $3174(31.7)$ & $3238(32.4)$ & $3192(31.9)$ & $3143(31.4)$ & $3195(32.0)$ \\
\hline 2 & $3379(33.8)$ & $3441(34.4)$ & $3368(33.7)$ & $3455(34.6)$ & $3465(34.7)$ \\
\hline \multicolumn{6}{|l|}{ No. of children (lifetime) } \\
\hline 0 & $200(2.0)$ & $205(2.1)$ & $196(2.0)$ & $205(2.1)$ & $197(2.0)$ \\
\hline 1 & $1783(17.8)$ & $1841(18.4)$ & $1827(18.3)$ & $1744(17.4)$ & $1710(17.1)$ \\
\hline \multirow[t]{2}{*}{2} & $8017(80.2)$ & $7954(79.5)$ & $7977(79.8)$ & $8051(80.5)$ & $8093(80.9)$ \\
\hline & \multicolumn{5}{|c|}{ Mean (standard-deviation) } \\
\hline $\begin{array}{l}\text { Interval time between the } \\
\text { first child and the second } \\
\text { child (years) }\end{array}$ & $7.40(5.24)$ & $7.28(5.10)$ & $7.40(5.24)$ & $7.35(5.20)$ & $7.34(5.22)$ \\
\hline
\end{tabular}

Notes: $e$ : prospective father: medium fertility intention, prospective mother: medium fertility intention;

$f$ : prospective father: medium fertility intention, prospective mother: low fertility intention;

$g$ : prospective father: low fertility intention, prospective mother: high fertility intention;

$h$ : prospective father: low fertility intention, prospective mother: medium fertility intention;

$i$ : prospective father: low fertility intention, prospective mother: low fertility intention.

the end of the five-year simulation, more than one third of households have two children. In addition, there is nearly no difference among control group and experimental plans' outcomes on the interval time between the first child and the second child.

In the Table $2 \mathrm{a}$ and Table $2 \mathrm{~b}$, we present the outcomes of experimental program II. Around $40 \%$ of households have no child in the end of the two-year simulation. In the end of the five-year simulation, more than one third of households have two children. Further, we can find that basically the more harmonious relations among family members, the higher ratio of families having the second child in the end of the lifetime simulation. 
Table 2a. Experiment II's outcomes of families' fertility behaviors under adjusting relationships between family members

\begin{tabular}{|c|c|c|c|c|}
\hline \multirow[t]{2}{*}{ Variable } & \multicolumn{4}{|c|}{$\begin{array}{l}\text { Experimental outcome: numbers of families (percent of } \\
\text { families) }\end{array}$} \\
\hline & $a$ & $b$ & $c$ & $d$ \\
\hline \multicolumn{5}{|l|}{ No. of children (2 years) } \\
\hline 0 & $3912(39.1)$ & $3887(38.9)$ & $3992(39.9)$ & $3949(39.5)$ \\
\hline 1 & $3567(35.7)$ & $3684(36.8)$ & $3547(35.5)$ & $3688(36.9)$ \\
\hline 2 & $2521(25.2)$ & $2429(24.3)$ & $2461(24.6)$ & $2363(23.6)$ \\
\hline \multicolumn{5}{|l|}{ No. of children (5 years) } \\
\hline 0 & $2920(29.2)$ & $2948(29.5)$ & $3056(30.6)$ & $3022(30.2)$ \\
\hline 1 & $3054(30.5)$ & $3162(31.6)$ & $3061(30.6)$ & $3140(31.4)$ \\
\hline 2 & $4026(40.3)$ & 3890 (38.9) & $3883(38.8)$ & $3838(38.4)$ \\
\hline \multicolumn{5}{|l|}{ No. of children (lifetime) } \\
\hline 0 & $853(8.5)$ & $879(8.8)$ & $939(9.4)$ & $885(8.9)$ \\
\hline 1 & $4994(49.9)$ & $5101(51.0)$ & $4950(49.5)$ & $4981(49.8)$ \\
\hline \multirow[t]{2}{*}{2} & $4153(41.5)$ & $4020(40.2)$ & $4111(41.1)$ & $4134(41.3)$ \\
\hline & \multicolumn{4}{|c|}{ Mean (standard-deviation) } \\
\hline $\begin{array}{l}\text { Interval time between the } \\
\text { first child and the second } \\
\text { child (years) }\end{array}$ & $5.70(4.57)$ & $5.71(4.55)$ & $5.77(4.43)$ & $5.74(4.56)$ \\
\hline
\end{tabular}

Notes: $a$ : couples' relationships: great; relations between couples and their own parents: great; relations between couples and their parents-in-law: great;

$b$ : couples' relationships: great; relations between couples and their own parents: great; relations between couples and their parents-in-law: normal;

$c$ : couples' relationships: great; relations between couples and their own parents: normal; relations between couples and their parents-in-law: great;

$d$ : couples' relationships: great; relations between couples and their own parents: normal; relations between couples and their parents-in-law: normal. 
Table 2b. Experiment II's outcomes of families' fertility behaviors under adjusting relationships between family members

\begin{tabular}{|c|c|c|c|c|}
\hline \multirow[t]{2}{*}{ Variable } & \multicolumn{4}{|c|}{$\begin{array}{l}\text { Experimental outcome: numbers of families (percent of } \\
\text { families) }\end{array}$} \\
\hline & $e$ & $f$ & $g$ & $h$ \\
\hline \multicolumn{5}{|l|}{ No. of children ( 2 years) } \\
\hline 0 & $4181(41.8)$ & $4197(42.0)$ & $4152(41.5)$ & $4137(41.4)$ \\
\hline 1 & $3641(36.4)$ & $3697(37.0)$ & $3673(36.7)$ & 3759 (37.6) \\
\hline 2 & $2178(21.8)$ & $2106(21.1)$ & $2175(21.8)$ & $2104(21.0)$ \\
\hline \multicolumn{5}{|l|}{ No. of children ( 5 years) } \\
\hline 0 & 3364 (33.6) & $3394(33.9)$ & 3389 (33.9) & $3391(33.9)$ \\
\hline 1 & $3141(31.4)$ & $3239(32.4)$ & $3109(31.1)$ & $3221(32.2)$ \\
\hline 2 & $3495(34.9)$ & $3367(33.7)$ & $3502(35.0)$ & $3388(33.9)$ \\
\hline \multicolumn{5}{|l|}{ No. of children (lifetime) } \\
\hline 0 & $1090(10.9)$ & $1147(11.5)$ & $1219(12.2)$ & $1183(11.8)$ \\
\hline 1 & $4884(48.8)$ & $4949(49.5)$ & $5027(50.3)$ & $5019(50.2)$ \\
\hline \multirow[t]{2}{*}{2} & $4026(40.3)$ & $3904(39.0)$ & $3754(37.5)$ & $3798(38.0)$ \\
\hline & \multicolumn{4}{|c|}{ Mean (standard-deviation) } \\
\hline $\begin{array}{l}\text { Interval time between the } \\
\text { first child and the second } \\
\text { child (years) }\end{array}$ & $5.98(4.78)$ & $6.03(4.68)$ & $5.99(4.66)$ & $5.90(4.58)$ \\
\hline
\end{tabular}

Notes: $e$ : couples' relationships: normal; relations between couples and their own parents: great; relations between couples and their parents-in-law: great;

$f$ : couples' relationships: normal; relations between couples and their own parents: great; relations between couples and their parents-in-law: normal;

g: couples' relationships: normal; relations between couples and their own parents: normal; relations between couples and their parents-in-law: great;

$h$ : couples' relationships: normal; relations between couples and their own parents: normal; relations between couples and their parents-in-law: normal.

\section{Discussion, Conclusion and Future Work}

To model the mutual influence among agents' preferences (such as intentions) and the transformation from agents' preferences (intentions) to their behaviors in groups, we built a multi-agent system $\left(\mathrm{MAS}_{\mathrm{IITIB}}\right)$ in the context of social networks. Further, we ran experiments and simulations for the study of the interaction of fertility intentions and the generation of fertility behaviors in families, from a micro perspective of individual interactions instead of conventional macro perspective of entire social system in Demography. Our agent-based model handles the complicated multiple influencing relationships (stronger or weaker, positive or negative) among individual members in families, and the transformation from family members' fertility intentions to prospective mothers' fertility behaviors with consideration of randomness. In our research, we designed a series of experimental programs, and monitored the dynamics of fertility intentions and behaviors, and particularly the number of new born children 
in the long-term. We hope that our results would contribute to discovering important factors that affect the fertility behavior and population growth under different policies (particularly under consideration of the policy changed from the one-child policy to the universal two-child policy in China) and help the government to adjust policies and strategies for the well-being of society. However, our work is not yet sufficient, as many other prospects remain. One aspect is about the advancements of the general model of influence among preferences and its transformation to behaviors:

- We express agents' preferences in a cardinal approach, assuming a normalized value as intention for each agent, and use the intention values of agents as probabilities for the generation of their behaviors to include randomness in the formulation of the transformation from preferences to behaviors. An ordinal approach to represent agents' preferences, particularly the preference ordering among multiple alternatives, could be tried in future work.

- We have not provided enough material to support the multi-agent system we designed to accurately characterize the dynamics of agents' preferences and behaviors due to mutual influence in groups in practice. In the future, the contributions of other disciplines such as logic and psychology, cognitive science and behavior science, particularly the empirical evidences in these fields, would be much valuable.

- We assumed a linear relation between agents' intentions and the generation of their behaviors: the higher an agent's intention is, the more possible it is to produce a behavior, and the return brought by the increase of intention is proportional. While in reality, there may be some thresholds of intentions for the generation of behaviors, which means the increase of intention within certain range may have very limited impacts, but once beyond a certain threshold, the return brought by the increase of intention will be much tremendous. The artificial neural network could be used to model the complicated psychical process under the transformation from intentions to behaviors of humans in future work, particularly making full use of all kinds of non-linear activation functions.

The other aspect is about the improvements of the agent-based modeling and simulation on fertility intention and behavior:

- We mainly modeled the impact of age on a prospective mother's fertility intention evolution, but have to admit that there are many other potential influential factors such as the income and expense, health condition, and balance with her life plan. From the economic (rational) perspective on fertility behavior, a utility-based approach may be recommended. In this approach, prospective parents may try to optimize the expected utility combing both assistance and care in the elderly period provided by their children and expense defrayed and energy consumed during the childhood of their children. Economists may encourage us to introduce to the agentbased model some economic variables.

- We assumed one usual household structure composed of a husband and a wife, both two (prospective) grandparents on each side, and several relatives and close friends on each side. But in real-world situations, there are more kinds of household structure, for instance, there are not four grandparents alive. More household 
structures can be designed in future work, particularly according to the result of the census of population.

- We mainly discussed the interaction of fertility intentions and the generation of fertility behaviors in families under the impact of population policy transition. While the fertility intention and behavior are closely related to cultural traditions which are hard to be measured from a computational perspective. For instance, in China, the effectiveness of the implementation of population polices are quite different in different areas. Therefore, the fertility intention and behavior are affected not only by state interventions. The interplay of political, economic and cultural factors can be discussed in future work.

Moreover, with certain adjustments, there are a lot of potentials to use our model in studying the mutual-influence among preferences and the transformation from preferences to behaviors with various group settings.

Acknowledgements. This study is supported by a National Natural Science Foundation of China Grant (71804006), a National Natural Science Foundation of China and European Research Council Cooperation and Exchange Grant (7161101045), and a UKRI's Global Challenge Research Fund (ES/P011055/1). An earlier version of this study has been presented in the local proceedings of the $19^{\text {th }}$ International Conference on Group Decision and Negotiation held at Loughborough, UK, thanks for the advices from reviewers and audiences.

\section{References}

1. Andrew, M., Meen, G.: Population structure and location choice: a study of London and South East England. Pap. Reg. Sci. 85(3), 401-419 (2006)

2. Booth, H.: Demographic forecasting: 1980-2005 in review. Int. J. Forecast. 22(3), 547-581 (2006)

3. Callick, R.: China relaxes its one-child policy. The Australian, 24 Jan 2007

4. Capuano, N., Chiclana, F., Fujita, H., Herrera-Viedma, E., Loia, V.: Fuzzy group decision making with incomplete information guided by social influence. IEEE Trans. Fuzzy Syst. 26 (3), 1704-1718 (2018)

5. Degroot, M.H.: Reaching a consensus. J. Am. Stat. Assoc. 69(345), 118-121 (1974)

6. Demarzo, P.M., Vayanos, D., Zwiebel, J.: Persuasion bias, social influence, and unidimensional opinions. Q. J. Econ. 118(3), 909-968 (2003)

7. Friedkin, N.E., Johnsen, E.C.: Social influence and opinions. J. Math. Soc. 15(3-4), $193-$ 206 (1990)

8. Friedkin, N.E., Johnsen, E.C.: Social positions in influence networks. Soc. Netw. 19(3), 209-222 (1997)

9. Golub, B., Jackson, M.O.: Naive learning in social networks and the wisdom of crowds. Am. Econ. J. Microecon. 2(1), 112-149 (2010)

10. Gu, B., Wang, F., Guo, Z., Zhang, E.: China's local and national fertility policies at the end of the twentieth century. Popul. Dev. Rev. 33(1), 129-148 (2007)

11. Guo, Z.: Changes in family households in China in 1990s. Paper presented at The Academic Conference on the 2000 Population Census in China, 28-31 March, Beijing (2003). (in Chinese) 
12. Grabisch, M., Rusinowska, A.: A model of influence based on aggregation function. Math. Soc. Sci. 66(3), 316-330 (2013)

13. Grabisch, M., Rusinowska, A.: A model of influence in a social network. Theory Decis. 69 (1), 69-96 (2010). https://doi.org/10.1007/s11238-008-9109-z

14. Grabisch, M., Rusinowska, A.: A model of influence with an ordered set of possible actions. Theory Decis. 69(4), 635-656 (2010). https://doi.org/10.1007/s11238-009-9150-6

15. Grabisch, M., Rusinowska, A.: Influence functions, followers and command games. Games Econ. Behav. 72(1), 123-138 (2011)

16. Grabisch, M., Rusinowska, A.: Measuring influence in command games. Soc. Choice Welfare 33(2), 177-209 (2009). https://doi.org/10.1007/s00355-008-0350-8

17. Grandi, U., Lorini, E., Perrussel, L.: Propositional opinion diffusion. In: Proceedings of the 14th International Conference on Autonomous Agents and Multiagent Systems, pp. 989-997 (2015)

18. Keilman, N., Pham, D.Q.: Predictive intervals for age-specific fertility. Eur. J. Popul. 16(1), 41-65 (2000). https://doi.org/10.1023/A:1006385413134

19. Luo, H., Meng, Q.: Multi-agent simulation of SC reform and national game. World Econ. Polit. 6, 136-155 (2013). (in Chinese)

20. Luo, H.: How to address multiple sources of influence in group decision-making? In: Morais, D.C., Carreras, A., de Almeida, A.T., Vetschera, R. (eds.) GDN 2019. LNBIP, vol. 351, pp. 17-32. Springer, Cham (2019). https://doi.org/10.1007/978-3-030-21711-2_2

21. Luo, H.: Individual, coalitional and structural influence in group decision-making. In: Torra, V., Narukawa, Y., Pasi, G., Viviani, M. (eds.) MDAI 2019. LNCS (LNAI), vol. 11676, pp. 77-91. Springer, Cham (2019). https://doi.org/10.1007/978-3-030-26773-5_7

22. Lutz, W.: Scenario analysis in population projection. International Institute for Applied Systems Analysis [IIASA], Laxenburg (1995)

23. Maran, A., Maudet, N., Pini, M.S., Rossi, F., Venable, K.B.: A framework for aggregating influenced CP-nets and its resistance to bribery. In: Proceedings of the Twenty-Seventh AAAI Conference on Artificial Intelligence, pp. 668-674 (2013)

24. Maudet, N., Pini, M.S., Venable, K.B., Rossi, F.: Influence and aggregation of preferences over combinatorial domains. In: Proceedings of the 11th International Conference on Autonomous Agents and Multiagent Systems, pp. 1313-1314 (2012)

25. National Health and Family Planning Commission of the PRC. Q\&A about the New TwoChild Policy. http://en.nhfpc.gov.cn/2015-11/06/c_45715.htm. Accessed 01 Nov 2018

26. Prez, L.G., Mata, F., Chiclana, F., Gang, K., Herrera-Viedma, E.: Modelling influence in group decision making. Soft. Comput. 20(4), 1653-1665 (2016). https://doi.org/10.1007/ s00500-015-2002-0

27. Salehi-Abari, A., Boutilier, C.: Empathetic social choice on social networks. In: Proceedings of the 13th International Conference on Autonomous Agents and Multiagent Systems, pp. 693-700 (2014)

28. Wang, F., Cai, Y., Gu, B.: Population, policy, and politics: how will history judge China's one-child policy? Popul. Dev. Rev. 38, 115-129 (2013)

29. Wang, Z., Dang, Y., Wang, Y.: A new grey Verhulst model and its application. In: Proceedings of the IEEE International Conference on Grey Systems and Intelligent Services (2007)

30. Zeng, Y., Therese, H.: The effects of China's universal two-child policy. The Lancet $\mathbf{3 8 8}$ (10054), 1930-1938 (2016)

31. Zhai, Z., Li, L., Chen, J.: Accumulated couples and extra births under the universal two-child policy. Popul. Res. 4, 35-51 (2016). (in Chinese)

32. Zheng, B.: Population ageing and the impacts of the universal two-child policy on China's socio-economy. Econ. Polit. Stud. 4(4), 434-453 (2016) 